\title{
Nonlinear Fokker-Planck Equation in the Model of Asset Returns
}

\author{
Alexander SHAPOVALOV ${ }^{\dagger \ddagger \S}$, Andrey TRIFONOV ${ }^{\ddagger \S}$ and Elena MASALOVA $\ddagger$ \\ † Tomsk State University, 36 Lenin Ave., 634050 Tomsk, Russia \\ E-mail: shpv@phys.tsu.ru \\ ‡ Tomsk Polytechnic University, 30 Lenin Ave., 634050 Tomsk, Russia \\ E-mail: trifonov@mph.phtd.tpu.edu.ru,eash@mail2000.ru \\ $\S$ Mathematical Physics Laboratory, Tomsk Polytechnic University, \\ 30 Lenin Ave., 634050 Tomsk, Russia
}

Received September 30, 2007, in final form March 26, 2008; Published online April 06, 2008

Original article is available at http://www.emis.de/journals/SIGMA/2008/038/

\begin{abstract}
The Fokker-Planck equation with diffusion coefficient quadratic in space variable, linear drift coefficient, and nonlocal nonlinearity term is considered in the framework of a model of analysis of asset returns at financial markets. For special cases of such a FokkerPlanck equation we describe a construction of exact solution of the Cauchy problem. In the general case, we construct the leading term of the Cauchy problem solution asymptotic in a formal small parameter in semiclassical approximation following the complex WKBMaslov method in the class of trajectory concentrated functions.
\end{abstract}

Key words: Fokker-Planck equation; semiclassical asymptotics; the Cauchy problem; nonlinear evolution operator; trajectory concentrated functions

2000 Mathematics Subject Classification: 35K55; 62M10; 91B28; 91B84

\section{Introduction}

Studies of observations available at financial markets reveal empirically stated statistical regularities of asset price changes whose distributions are of broad interest providing more reasons to control of losses and gains in the course of quotations. Asset returns can be measured in terms of increments $\Delta \xi=\xi(t+\Delta t)-\xi(t)$ over the time interval $\Delta t$ of a time series $\xi(t)$ of quotations of an asset. The quantitative handling of high-frequency data of currency exchange rates (see [1] and references therein) have substantiated heavy tailed probability density functions of price increments $\Delta \xi$ for small time steps $\Delta t$, asymptotic power law (or heavy tails) of return distributions different from the Gaussian distribution, volatility clustering, etc.

Power law is considered to be well-established fact (see, e.g., [2]). Several semi-empirical mathematical models have been proposed to describe the non-Gaussian properties of asset return distributions. The most known of them are ARCH (auto regression conditional heteroskedastic models with time changing conditional variances) [3] and its modification GARCH [4], "truncated" Levy distributions [5], multiplicative noise models (see, e.g., [6]), multifractal cascade models with the so called HARCH effect [7].

The model proposed in [1] to describe asset return distributions uses the approach based on the Fokker-Planck equation (FPE) with variable coefficients. In some papers this equation is called the Fokker-Planck-Kolmogorov equation. The idea of the work [1] is based on similarity observation between asset return distributions and distributions of vortex velocity increments in turbulent cascades in hydrodynamics. This observation is verified by quantitative analysis 
of distribution parameters that justifies an analogy between turbulent cascade in a stream of liquid and information cascade at stock markets.

Following this semi-empirical analogy, we fix our attention on the power law of the probability density function (PDF) on one hand, and on the analogy with the turbulent cascade, on the other. For such a system, self-organization phenomena are the characteristics that could be mathematically expressed by an additional nonlinear mean drift term in the FPE [8].

In this work we modify the FPE with the diffusion coefficient quadratic in $\Delta \xi$ considered in [1] by introducing the nonlinear mean drift term. For such a FPE, we apply the approach developed in $[9,10,11]$ which reduces the integrability problem for the nonlinear FPE to the problem for a linear equation associated with the original nonlinear FPE. We construct the exact solution of the nonlinear FPE whose coefficients have a special form, and for the coefficients of a general form we construct the leading term of solution asymptotic in a formal small parameter in semiclassical approximation in the class of trajectory concentrated functions.

\section{Formalism of the Fokker-Planck equation}

The formalism, proposed in [1] to describe distributions of stock market returns which asymptotically obey power law, is based on the Fokker-Planck equation in the evolution variable $\Delta t$. The power law exponents are determined from the Kramers-Moyal coefficients founded in the framework of a phenomenological method developed in [1]. The FPE provides the information as to how the distributions on different time intervals $\Delta t$ are correlated. In view of the analogy with the turbulent cascade the FPE is written in [1] in terms of logarithmic time-scale cascade proceeds.

The FPE for the probability density function $u(\Delta \xi, \tau)$ of price increment $\Delta \xi$ at a given logarithmic time scale $\tau$ is derived in [1] in the standard way from the Chapman-Kolmogorov equation and is presented in the form

$$
\partial_{\tau} u(\Delta \xi, \tau)=\left[-\frac{\partial}{\partial(\Delta \xi)} D^{(1)}(\Delta \xi, \tau)+\frac{\partial^{2}}{\partial(\Delta \xi)^{2}} D^{(2)}(\Delta \xi, \tau)\right] u(\Delta \xi, \tau)
$$

Here $\partial_{\tau}=\partial / \partial \tau$, and the Kramers-Moyal coefficients, the drift $D^{(1)}(\Delta \xi, \tau)$ and diffusion $D^{(2)}(\Delta \xi, \tau)$ coefficients, are found directly from the financial data and the following approximations were obtained

$$
\begin{aligned}
& D^{(1)}(\Delta \xi, \tau)=-0.44 \Delta \xi \\
& D^{(2)}(\Delta \xi, \tau)=0.003 e^{-\tau / 2}+0.019(\Delta \xi+0.04)^{2} .
\end{aligned}
$$

We assume the solutions $u(\Delta \xi, \tau)$ of equation (1) to be real smooth functions decreasing as $|\Delta \xi| \rightarrow \infty$. Notice, that equation (1) can be written as a balance relation that makes obvious the conservation of the integral $\int_{-\infty}^{+\infty} u(\Delta \xi, \tau) d(\Delta \xi)$. Taking the initial function $u(\Delta \xi, 0)$ to be normalized, we have

$$
\int_{-\infty}^{+\infty} u(\Delta \xi, \tau) d(\Delta \xi)=1, \quad \tau \geqslant 0
$$

The linear FPE (1), (2), (3) is solved numerically in [1] and quantitative agreement is obtained between the solutions and distributions constructed directly from the data of quotations, i.e. experimental data.

A more detailed analysis of the power laws $u(\Delta \xi) \sim(\Delta \xi)^{-(1+\mu)}$ made in [12] shows that the exponents $\mu$ found in the framework of the FPE formalism are not in good agreement with experimentally obtained values. 
Different ideas have been involved in studying of "heavy tails" of financial time-series distributions. Mathematically, such nonlinear stochastic systems are described in terms of the Fokker-Planck equation with coefficients dependent on the distribution. We consider the feedback caused by reciprocal effects of assets at the financial market. In accordance with [8] and by analogy between the exchange rates and the hydrodynamic turbulence $[1,12]$ let us modify the FPE (1) assuming that its coefficients $D^{(1)}$ and $D^{(2)}$ depend on the order parameter described by the first moment

$$
X_{u}(\tau)=\int_{-\infty}^{\infty} \Delta \xi u(\Delta \xi, \tau) d(\Delta \xi)
$$

In this work we consider analytical methods of solution construction for the following special case of the nonlinear Fokker-Planck equation (NFPE)

$$
\partial_{\tau} u(\Delta \xi, \tau)=\left[\frac{\partial}{\partial(\Delta \xi)}\left(\alpha \Delta \xi+\varkappa X_{u}(\tau)\right)+\frac{\partial}{\partial(\Delta \xi)} D^{(2)}(\Delta \xi, \tau) \frac{\partial}{\partial(\Delta \xi)}\right] u(\Delta \xi, \tau) .
$$

Here $\alpha$ and $\varkappa$ are real positive parameters. The diffusion coefficient $D^{(2)}$ is taken in more general form as compared to (3), i.e.

$$
D^{(2)}(\Delta \xi, \tau)=\epsilon\left(f(\tau)+(a \Delta \xi+g(\tau))^{2}\right) .
$$

Here $f(\tau)(\geqslant 0)$ and $g(\tau)$ are real smooth functions, $a$ is a real parameter, $\epsilon$ is a real positive parameter.

Market impact on price increment $\Delta \xi$ of an asset with the probability density function $u(\Delta \xi, \tau)$ determined by $(6)$, is modeled by the coefficient $\varkappa X_{u}(\tau)$.

The motivation of the drift coefficient

$$
D^{(1)}(\Delta \xi, \tau)=-\alpha \Delta \xi-\varkappa X_{u}(\tau)
$$

in (6) can be taken from the stochastic methods [14] and empirical data handling $[1,12]$. In the Fokker-Planck equation

$$
\partial_{\tau} u(\Delta \xi, \tau)=\partial_{\Delta \xi}\left[-V_{\Delta \xi}(\Delta \xi, \tau)+D^{(2)} \partial_{\Delta \xi}\right] u(\Delta \xi, \tau)
$$

the function $V(\Delta \xi, \tau)$ can be treated as a potential of a regular external force $V_{\Delta \xi}(\Delta \xi, \tau)=$ $\partial_{\Delta \xi} V(\Delta \xi, \tau)$ [14]. Let us assume that there are a number of mutually interacting assets on the financial market. Following the mean field concept [8], we modify the FPE (9) by substitution

$$
V_{\Delta \xi}(\Delta \xi, \tau) \rightarrow V_{\Delta \xi}(\Delta \xi, \tau)+\int_{-\infty}^{\infty} W_{\Delta \xi}(\Delta \xi-y, \tau) u(y, \tau) d y
$$

Here the integral simulates the cumulative effect of other market assets on price increment $\Delta \xi$ of a separate asset with a potential $W(\Delta \xi-y, \tau)$ which depends on the difference $\Delta \xi-y$ due to symmetry of mutual asset effects.

In accordance with $[1,12]$, where linear form of the drift $V_{\Delta \xi}(\Delta \xi, \tau)=\tilde{\alpha} \Delta \xi$ gives close approximations of empirical data, we take modified drift coefficient (10) as $-\tilde{\alpha} \Delta \xi+\varkappa \int_{-\infty}^{\infty}(\Delta \xi-$ y) $u(y, \tau) d y$ that results in $-\alpha \Delta \xi-\varkappa X_{u}(\tau), \alpha=\tilde{\alpha}-\varkappa$, that, in turn, gives (8).

To estimate the parameter $\varkappa$, one can take into account that $\varkappa X_{u}(\tau)$ in $(8)$ is responsible for cumulative effect of all marketable assets on the price increment $\Delta \xi$ of a given asset. It is assumed that in the stock market $\varkappa X_{u}(\tau)$ can be estimated with the help of an appropriate market index, as it characterizes the market as a whole. In the exchange operations (FOREX market), where indices are not used, such a characteristics could be deduced from the correlation 
matrix of currency exchange rates. Anyway, the estimation of the parameter $\varkappa$ is a subject of a special study.

The integrability problem for the FPE in applications is practically solved numerically. As a rule, analytical approaches are based on special classes of functions in which solutions are constructed. A more detailed review of solution methods for the NFPE (6) can be found in [11].

Using the approach considered in [11] we obtain the evolution operator for equation (6) with a constant diffusion coefficient $D^{(2)}=$ const. This result is used to construct exact solutions of (6) for the functions $f(\tau)$ and $g(\tau)$ of a special form. Following [10] for these functions of general form, we construct asymptotic solutions of (6) in semiclassical approximation in a small parameter $\epsilon \rightarrow 0$ in the class of trajectory concentrated functions.

\section{Evolution operator for the FPE with constant diffusion}

Let us assume that in the NFPE (6) the diffusion is constant, $D^{(2)}(\Delta x, \tau)=\epsilon$. Setting $\Delta \xi=x$ for convenience, we rewrite (6) as

$$
\partial_{\tau} u(x, \tau)=\left[\partial_{x}\left(\alpha x+\varkappa X_{u}(\tau)\right)+\epsilon \partial_{x x}\right] u(x, \tau),
$$

where $\partial_{x}=\partial / \partial x, \partial_{x x}=\partial^{2} /(\partial x)^{2}$. Equation (11) plays the basic role in our study of the nonlinear Fokker-Planck equation $(6)$ and in the linear case $(\varkappa=0)$ it describes the OrnsteinUhlenbeck process [16] (see also [14]).

Let us take a fixed value $\tau=s$ as the initial time and consider the Cauchy problem

$$
u(x, s)=\gamma(x)
$$

for equation (11) in a class of functions of the Schwartz space $\mathcal{S}$ in the variable $x$ for $\tau \geqslant s$. In accordance with (4) we can set

$$
\gamma(x) \in \mathcal{S}, \quad \int_{-\infty}^{+\infty} \gamma(x) d x=1
$$

without the loss of generality then

$$
\int_{-\infty}^{+\infty} u(x, \tau) d x=1, \quad \tau \geqslant s
$$

For the first moment (5) of the function $u(x, \tau)$,

$$
X_{u}(\tau)=\int_{-\infty}^{+\infty} x u(x, \tau) d x
$$

from (11) and (13) we immediately obtain

$$
\dot{X}_{u}(\tau)=-(\alpha+\varkappa) X_{u}(\tau)
$$

Here $\dot{X}_{u}(\tau)=d X_{u}(\tau) / d \tau$, then

$$
\begin{aligned}
& X_{u}(\tau)=X_{\gamma} \exp [-(\alpha+\varkappa)(\tau-s)], \\
& X_{\gamma}=\int_{-\infty}^{+\infty} x \gamma(x) d x .
\end{aligned}
$$

Therefore, the first moment $X_{u}(\tau)$ is determined by the initial distribution $\gamma(x)$ and does not require the solution of equation (11). The substitution of (16) into (11) actually linearizes the 
NFPE. Such a property of the NFPE with coefficients depending on the first moment $X_{u}(\tau)$ is related to the fact that this equation is close to the linear one (see [11] for more details).

Let us present the solution of the Cauchy problem (11) and (12) as action of the evolution operator $\widehat{U}(\tau, s, \cdot)$ on the initial function $\gamma(x)$. Following [11] we obtain

$$
u(x, \tau)=\widehat{U}(\tau, s, \gamma)(x)=\int_{-\infty}^{+\infty} G_{\operatorname{lin}}\left(\tau, s, x-X_{u}(\tau), y-X_{\gamma}\right) \gamma(y) d y
$$

Here $G_{\operatorname{lin}}(\tau, s, x, y)$ is the Green function of the linear FPE resulting from (11) with the assumption $\varkappa=0$,

$$
G_{\operatorname{lin}}(\tau, s, x, y)=\frac{e^{\frac{\alpha}{2}(\tau-s)}}{\sqrt{\frac{4 \pi \epsilon}{\alpha} \sinh \alpha(\tau-s)}} \exp \left[-\frac{\alpha e^{\alpha(\tau-s)}}{4 \epsilon \sinh \alpha(\tau-s)}\left(x-e^{-\alpha(\tau-s)} y\right)^{2}\right] .
$$

Notice that the solution of the Cauchy problem (11) and (12) can be immediately generalized for the multidimensional FPE.

\section{Nonlinear FPE with quadratic diffusion}

Consider the NFPE

$$
\partial_{\tau} u(x, \tau)=\left[\partial_{x}(\alpha x+\varkappa \beta(\tau))+\epsilon \partial_{x}\left(f(\tau)+(a x+g(\tau))^{2}\right) \partial_{x}\right] u(x, \tau),
$$

where coefficients $\beta(\tau), f(\tau)$, and $g(\tau)$ in general case can depend on $X_{u}(\tau)$.

With the obvious notation $\Delta \xi=x$, equation (19) takes the form (6), (7) when $\beta(\tau)=X_{u}(\tau)$, and $f(\tau)$ and $g(\tau)$ do not depend on $X_{u}(\tau)$, and, therefore, (19) generalizes (6), (7).

From (13), (14), and (19) by analogy with (15) we immediately obtain

$$
\dot{X}_{u}(\tau)=-\left(\alpha-2 a^{2} \epsilon\right) X_{u}(\tau)-\varkappa \beta(\tau)+2 a \epsilon g(\tau) .
$$

Similarly to equation (11), the Cauchy problem (12) for the NFPE (19) induces the Cauchy problem (20) and

$$
X_{u}(s)=X_{\gamma}
$$

determining $X_{u}(\tau)$ independently of the solution of the FPE (19), where $X_{\gamma}$ has the form (17). When $g(\tau)$ does not depend on $X_{u}(\tau)$ and $\beta(\tau)=X_{u}(\tau)$, we have the following explicit expression for $X_{u}(\tau)$ from $(20)$ and $(21)$

$$
X_{u}(\tau)=e^{-\left(\alpha+\varkappa-2 a^{2} \epsilon\right)(\tau-s)} X_{\gamma}+2 a \epsilon \int_{s}^{\tau} e^{-\left(\alpha+\varkappa-2 a^{2} \epsilon\right)(\tau-\xi)} g(\xi) d \xi .
$$

The solution of equation (20) can be found also for a different form of dependence of $g(\tau)$ on $X_{u}(\tau)$. In any case, finding of the moment $X_{u}(\tau)$ separately from solution of the nonlinear FPE and substitution $X_{u}(\tau)$ into equation (19) actually linearizes this equation with respect to the PDF $u(x, \tau)$.

In order to derive exact and approximate solutions it is helpful to introduce new variables $(y, \tau)$,

$$
y=\frac{1}{a} \log \left(a x+g(\tau)+\sqrt{f(\tau)+(a x+g(\tau))^{2}}\right),
$$


such that in the new variables the diffusion term becomes a constant. In the new variables equation (19) takes the form

$$
\partial_{\tau} u(y, \tau)=\left[\alpha+\frac{q(y, \tau)}{A_{(+)}(y, \tau)} \partial_{y}+\epsilon \partial_{y y}\right] u(y, \tau) .
$$

Here the following notations are used:

$$
\begin{aligned}
& q(y, \tau, \epsilon)=2\left(\varkappa \beta(\tau)-\frac{\alpha}{a} g(\tau)-\frac{\dot{g}(\tau)}{a}\right)-\frac{\dot{f}(\tau)}{a} e^{-a y}+\left(\frac{\alpha}{a}+a \epsilon\right) A_{(-)}(y, \tau), \\
& A_{( \pm)}(y, \tau)=e^{a y} \pm f(\tau) e^{-a y} .
\end{aligned}
$$

Let us impose special constraints on the functions $\beta(\tau), g(\tau)$, and $f(\tau)$ to reduce $(23)$ to a solvable form and to construct exact solutions.

\subsection{Exact solution}

An exact solution of equation (19) can be found if the functions $f(\tau)$ and $g(\tau)$ obey the conditions

$$
\dot{f}(\tau)+2 a c f(\tau)=0, \quad \dot{g}(\tau)+\alpha g(\tau)-a \varkappa \beta(\tau)=0, \quad c=\frac{\alpha}{a}+a \epsilon .
$$

In this case $f(\tau)=f(s) e^{-2 a c(\tau-s)}, g(\tau)=g(s) e^{-\alpha(\tau-s)}+a \varkappa \int_{s}^{\tau} e^{-\alpha(\tau-\xi)} \beta(\xi) d \xi$, and equation (23) takes the form

$$
\left[-\partial_{\tau}+c \partial_{y}+\alpha+\epsilon \partial_{y y}\right] u(y, \tau)=0 .
$$

By changing the variables $(y, \tau) \rightarrow(z, \tau)$, where $z=y+c \tau$ and taking $v(z, \tau)=u(y, \tau) e^{-\alpha \tau}$, we reduce $(27)$ to the standard diffusion equation

$$
\left[-\partial_{\tau}+\epsilon \partial_{z z}\right] v(z, \tau)=0,
$$

whose Green function is well known

$$
G_{s t}\left(\tau, s, z, z^{\prime}\right)=\frac{1}{\sqrt{4 \pi \epsilon(\tau-s)}} e^{-\frac{\left(z-z^{\prime}\right)^{2}}{4 \epsilon(\tau-s)}}
$$

where a fixed value $\tau=s$ is taken as the initial time.

Then, for equation (27) we have

$$
G\left(\tau, s, y, y^{\prime}\right)=\frac{e^{\alpha(\tau-s)}}{\sqrt{4 \pi \epsilon(\tau-s)}} \exp \left\{-\frac{1}{4 \epsilon(\tau-s)}\left[y-y^{\prime}+c(\tau-s)\right]^{2}\right\},
$$

and for equation (19)

$$
\begin{aligned}
G\left(\tau, s, x, x^{\prime}\right)= & \frac{e^{\alpha(\tau-s)}}{\sqrt{4 \pi \epsilon(\tau-s)}} \exp \left\{-\frac{1}{4 \epsilon(\tau-s)}\left[\frac{1}{a} \log \frac{a x+g(\tau)+\sqrt{f(\tau)+(a x+g(\tau))^{2}}}{a x^{\prime}+g(s)+\sqrt{f(s)+\left(a x^{\prime}+g(s)\right)^{2}}}\right.\right. \\
& \left.+c(\tau-s)]^{2}\right\}
\end{aligned}
$$

when conditions (26) are satisfied. Notice that the Green function (30) can be considered as kernel of the correspondent evolution operator of the NFPE (19), i.e.

$$
u(x, \tau)=\widehat{U}(\tau, s, \gamma)(x)=\int_{-\infty}^{+\infty} G\left(\tau, s, x, x^{\prime}\right)\left(f(\tau)+\left(a x^{\prime}+g(\tau)\right)^{2}\right)^{-1 / 2} \gamma\left(x^{\prime}\right) d x^{\prime} .
$$

The evolution operator $\widehat{U}$ is nonlinear because the functions $\beta(\tau), f(\tau)$ and $g(\tau)$ entering the Green function (30) depend on the function $u\left(x^{\prime}, s\right)=\gamma\left(x^{\prime}\right)$ with the operator $\widehat{U}$ acting on it. 


\subsection{Semiclassical approximation}

For equation (23) with the coefficients $\beta(\tau), f(\tau)$, and $g(\tau)$ that are generally dependent on $\tau$ but independent of $\epsilon$ we construct a leading term of formal solution of the Cauchy problem and the evolution operator asymptotic in small parameter $\epsilon(\epsilon \rightarrow 0)$ in the class of trajectoryconcentrated functions.

Let us introduce class $\mathcal{P}_{\epsilon}^{\tau}(Y(\tau, \epsilon))$ of functions

$$
\Phi(y, \tau, \epsilon)=\varphi\left(\frac{\Delta y}{\sqrt{\epsilon}}, \tau, \epsilon\right)
$$

where $\Delta y=y-Y(\tau, \epsilon), y$ is given by $(22)$, and $Y(\tau, \epsilon)$ is a functional parameter of the class, regularly depends on $\epsilon$ and is to be determined. The function $\varphi(\xi, \tau, \epsilon)$ belongs to the Schwartz space, smoothly depends on $\xi$ and $\tau$, decreases with $\xi \rightarrow \infty$, and regularly depends on $\epsilon$ for $\epsilon \rightarrow 0$, i.e. it can be expanded in the Taylor series around $\epsilon=0$.

Functions of the class $\mathcal{P}_{\epsilon}^{\tau}(Y(\tau, \epsilon))$ are normalized with respect to the norm

$$
\|\Phi\|^{2}(\tau, \epsilon)=\int_{\mathbb{R}^{1}} \Phi^{2}(\xi, \tau, \epsilon) d \xi
$$

By analogy with $[10,15]$ let us call solutions of the class $\mathcal{P}_{\epsilon}^{\tau}(Y(\tau, \epsilon))$ the trajectory concentrated solutions. Restrict ourself with the case when $Y(\tau, \epsilon)$ does not depend on $\epsilon, Y(\tau, \epsilon)=Y(\tau)$. We are interested here in solutions of equation (23) localized in $y$ in a neighborhood of the curve $y=Y(\tau)$ for each fixed $\epsilon \in\left[0,1\left[\right.\right.$ and $\tau \in \mathbb{R}^{1}$.

For the functions belonging to $\mathcal{P}_{\epsilon}^{\tau}(Y(\tau))$, the following asymptotic estimates can be immediately obtained

$$
\begin{aligned}
& \frac{\left\|(\Delta y)^{k} \Phi\right\|(\tau, \epsilon)}{\|\Phi\|(\tau, \epsilon)}=O\left(\epsilon^{k / 2}\right), \quad \frac{\left\|\left(\epsilon \partial_{y}\right)^{k} \Phi\right\|(\tau, \epsilon)}{\|\Phi\|(\tau, \epsilon)}=O\left(\epsilon^{k / 2}\right), \\
& \frac{\left\|\epsilon\left(\partial_{\tau}+\dot{Y}(\tau, \epsilon) \partial_{y}\right) \Phi\right\|(\tau, \epsilon)}{\|\Phi\|(\tau, \epsilon)}=O(\epsilon) .
\end{aligned}
$$

Notice that $\xi=\frac{y-Y(\tau)}{\sqrt{\epsilon}}$ has the estimate $\xi \sim O(1)$.

Transformation from $(y, \tau)$ to the variables $(\xi, \tau)$ reduces equation $(23)$ to the form

$$
\widehat{L} \varphi(\xi, \tau, \epsilon)=\left[-\partial_{\tau}+\alpha+\left(\dot{Y}(\tau)+\frac{q(\sqrt{\epsilon} \xi+Y(\tau), \tau)}{A_{(+)}(\sqrt{\epsilon} \xi+Y(\tau), \tau)}\right) \frac{1}{\sqrt{\epsilon}} \partial_{\xi}+\partial_{\xi \xi}\right] \varphi(\xi, \tau, \epsilon)=0,
$$

where notations (24), (25) are used.

Let us expand coefficients of equation $(31)$ and the function $\varphi(\xi, \tau, \epsilon)$ into power series in $\sqrt{\epsilon}$, e.g.

$$
\varphi(\xi, \tau, \epsilon)=\varphi^{(0)}(\xi, \tau)+\sqrt{\epsilon} \varphi^{(1)}(\xi, \tau)+\cdots
$$

Equating coefficients at equal powers of $(\sqrt{\epsilon})^{k / 2}, k=0,1,2, \ldots$, in (27) we obtain the following equations determining asymptotic solution of equation (31)

$$
\begin{aligned}
& \dot{Y}(\tau)+\frac{q_{0}(Y(\tau), \tau)}{A_{(+)}(Y(\tau), \tau)}=0, \\
& \widehat{L}_{(0)} \varphi^{(0)}(\xi, \tau)=0, \\
& \widehat{L}_{(0)} \varphi^{(1)}(\xi, \tau)=-\widehat{L}_{(1)} \varphi^{(0)}(\xi, \tau) .
\end{aligned}
$$


Here we use the notations

$$
\begin{aligned}
\hat{L}_{(0)}= & -\partial_{\tau}+\partial_{\xi \xi}+\xi R(\tau) \partial_{\xi}+\alpha, \\
\hat{L}_{(1)}= & \left(Q_{0}(\tau) \xi^{2}+Q_{1}(\tau)\right) \partial_{\xi}, \\
R(\tau)= & \frac{1}{A_{(+)}(Y(\tau), \tau)}\left[\dot{f}(\tau) e^{-a Y(\tau)}+\alpha A_{(+)}(Y(\tau), \tau)+a \dot{Y}(\tau) A_{(-)}(Y(\tau), \tau)\right], \\
Q_{0}(\tau)= & \frac{a}{2 A_{(+)}(Y(\tau), \tau)}\left[-\dot{f}(\tau) e^{-a Y(\tau)}+\alpha A_{(-)}(Y(\tau), \tau)+a \dot{Y}(\tau) A_{(+)}(Y(\tau), \tau)\right] \\
& \quad-\frac{a A_{(-)}(Y(\tau), \tau)}{A_{(+)}(Y(\tau), \tau)} R(\tau), \quad Q_{1}(\tau)=\frac{a A_{(-)}(Y(\tau), \tau)}{A_{(+)}(Y(\tau), \tau)}, \\
q_{0}(y, \tau)= & \left.q(y, \tau, \epsilon)\right|_{\epsilon=0}=2\left(\varkappa \beta(\tau)-\frac{\alpha}{a} g(\tau)-\frac{\dot{g}(\tau)}{a}\right)-\frac{\dot{f}(\tau)}{a} e^{-a y}+\frac{\alpha}{a} A_{(-)}(y, \tau) .
\end{aligned}
$$

It is obvious that the construction of the asymptotic solution is reduced to the solution of equation (34).

By changing dependent and independent variables in (34) according to the formulas

$$
\begin{aligned}
& \varphi^{(0)}(\xi, \tau)=e^{\alpha(\tau-s)} v\left(z, \tau^{\prime}\right), \quad \tau^{\prime}=\int_{s}^{\tau} h^{2}(\eta) d \eta, \\
& z=h(\tau) \xi, \quad h(\tau)=h(s) \exp \left(\int_{s}^{\tau} R(\eta) d \eta\right),
\end{aligned}
$$

we reduce equation (34) to equation (28) with $\epsilon=1$ whose Green function is given by (29), i.e.

$$
G_{s t}\left(\tau^{\prime}, 0, z, z^{\prime}\right)=\frac{1}{\sqrt{4 \pi \tau^{\prime}}} e^{-\frac{\left(z-z^{\prime}\right)^{2}}{4 \tau^{\prime}}} .
$$

Notice that in (36) $\tau=s$ corresponds to $\tau^{\prime}=0$. Therefore, the solution of the Cauchy problem $\left(-\partial_{\tau^{\prime}}+\partial_{z z}\right) v\left(z, \tau^{\prime}\right)=0, v(z, 0)=v_{0}(z)$ given by the Green function (37) reads

$$
v\left(z, \tau^{\prime}\right)=\int_{-\infty}^{\infty} G_{s t}\left(\tau^{\prime}, 0, z, z^{\prime}\right) v_{0}\left(z^{\prime}\right) d z^{\prime}
$$

and for the problem $\left(-\partial_{\tau^{\prime}}+\partial_{z z}\right) u\left(z, \tau^{\prime}\right)=F\left(z, \tau^{\prime}\right), u(z, 0)=0$, we have

$$
u\left(z, \tau^{\prime}\right)=\int_{0}^{\tau^{\prime}} d \eta \int_{-\infty}^{\infty} G_{s t}\left(\tau^{\prime}-\eta, 0, z, z^{\prime}\right) F\left(z^{\prime}, \eta\right) d z^{\prime}
$$

Let us now find the asymptotic solution of the Cauchy problem

$$
\varphi(\xi, s)=\gamma(\xi)
$$

for equation (31) in the form (32). To this end we change variables from $\left(z, \tau^{\prime}\right)$ to $(\xi, \tau)$ in $(38)$ according to (36) and obtain the solution of the Cauchy problem $\varphi^{(0)}(\xi, s)=\gamma(\xi)$ for equation $(34)$

$$
\begin{aligned}
\varphi^{(0)}(\xi, \tau) & =\int_{-\infty}^{\infty} e^{\alpha(\tau-s)} G_{s t}\left(\tau^{\prime}(\tau), 0, h(\tau) \xi, h(s) \xi^{\prime}\right) \gamma\left(\xi^{\prime}\right) h(s) d \xi^{\prime} \\
& =\int_{-\infty}^{\infty} \frac{e^{\alpha(\tau-s)}}{\sqrt{4 \pi \int_{s}^{\tau} h^{2}(\eta) d \eta}} \exp \left[-\frac{\left(h(\tau) \xi-h(s) \xi^{\prime}\right)^{2}}{4 \int_{s}^{\tau} h^{2}(\eta) d \eta}\right] \gamma\left(\xi^{\prime}\right) h(s) d \xi^{\prime}
\end{aligned}
$$


Here the relations $z^{\prime}=h(s) \xi^{\prime}, \gamma\left(\xi^{\prime}\right)=v_{0}\left(z^{\prime}\right)=v_{0}\left(h(s) \xi^{\prime}\right)$ are used. Similarly, in accordance with (39) for the function $\varphi^{(1)}(\xi, \tau)$ determined by (35) we have

$$
\varphi^{(1)}(\xi, \tau)=\int_{s}^{\tau} d \eta \int_{-\infty}^{\infty} e^{\alpha(\tau-\eta)} G_{s t}\left(\tau^{\prime}(\tau)-\tau^{\prime}(\eta), 0, h(\tau) \xi, h(\eta) \xi^{\prime}\right) \tilde{F}\left(\xi^{\prime}, \eta\right) h(\eta) d \xi^{\prime}
$$

where $F\left(h(\eta) \xi^{\prime}, \tau^{\prime}(\eta)\right)=e^{-\alpha(\eta-s)} h^{-2}(\eta) \tilde{F}\left(\xi^{\prime}, \eta\right), \tilde{F}(\xi, \tau)=-\hat{L}_{(1)} \varphi^{(0)}(\xi, \tau)$,

$$
\varphi^{(1)}(\xi, \tau)=\int_{s}^{\tau} d \eta \int_{-\infty}^{\infty} \frac{e^{\alpha(\tau-\eta)}}{\sqrt{4 \pi \int_{\eta}^{\tau} h^{2}(y) d y}} \exp \left[-\frac{\left(h(\tau) \xi-h(\eta) \xi^{\prime}\right)^{2}}{4 \int_{\eta}^{\tau} h^{2}(y) d y}\right] \tilde{F}\left(\xi^{\prime}, \eta\right) h(\eta) d \xi^{\prime}
$$

Thus, the function (32) determined by expressions (33), (41), (42) gives the asymptotic solution of the Cauchy problem (31), (40) accurate to $O(\epsilon)$ in the following meaning. Denote the action of the operator $\widehat{L}$ defined by (31) on the function $\varphi^{(0)}(\xi, \tau)+\sqrt{\epsilon} \varphi^{(1)}(\xi, \tau)$ as

$$
g^{(2)}(\xi, \tau, \epsilon):=\widehat{L}\left(\varphi^{(0)}(\xi, \tau)+\sqrt{\epsilon} \varphi^{(1)}(\xi, \tau)\right)
$$

then

$$
\frac{\left\|g^{(2)}\right\|(\tau, \epsilon)}{\|\varphi\|(\tau, \epsilon)}=O(\epsilon)
$$

Similarly to (18), we can consider the solution of the Cauchy problem as action of the evolution operator of the operator $\widehat{L}$ on the function $\gamma(\xi)$.

\section{Concluding remarks}

Asset interactions at the market are naturally supposed to be complex and nonlinear. The approach discussed in the work is intended for the investigation of the asset market by using simple nonlinear model directly generalizing known models based on the linear FPE (1). The asymptotics obtained, equations (32), (41), (43), can be considered as a necessary step in studying of nonlinear properties of the asset market.

The approach provides the way to analyze effects of nonlinearity on the distribution dynamics of asset price increments at the stock market or currency exchange. Such an analysis necessarily implies the statistical processing of corresponding empirical data to estimate values of the model parameters, which is beyond the scope of the present work and is a subject of a special research.

Following $[1,12,13]$, we use quadratic approximation for the diffusion coefficient (7) and linear drift (8). Our formalism can be also applied to the NFPE with more general dependence of coefficients on $\Delta \xi$. The quadratic approximation of the diffusion coefficient (7) is a generalization of the constant diffusion that results in non-Gaussian probability density functions. These PDFs are asymptotic solutions of the NFPE (19) in semiclassical approximation in explicit form given by (32), (41), (43) accurate to $O(\epsilon)$ in accordance with (44), (45). The construction of semiclassical solutions is based on exact solution of the NFPE with constant diffusion (11).

Preliminary calculations show that for the initial function $\gamma(x)$ having the power law asymptotics ("fat tails") the distribution keeps the non-Gaussian shape during its evolution.

In conclusion we would notice that since the stochastic properties of asset price increments are studied effectively by numerical simulation, it is of interest to formulate stochastic differential equations with the nonlinear feedback related to the NFPE considered above. 


\section{Acknowledgements}

The work was supported by the President of the Russian Federation, Grant No SS-871.2008.2 and Grant of the RFBR No 07-01-08035. A. Shapovalov acknowledges the partial support from the International Mathematical Union for participation in the Seventh International Conference "Symmetry in Nonlinear Mathematical Physics".

\section{References}

[1] Friedrich R., Peinke J., Renner Ch., How to quantify deterministic and random influence on the statistics of the foreign exchange market, Phys. Rev. Lett. 84 (2000), 5224-5227, physics/9901034.

[2] Mantegna R.N., Stanley H.E., An introduction to econophysics. Correlations and complexity in finance, London, Cambridge University Pres, 2000.

[3] Engle R., Autoregressive conditional heteroscedasticity with estimates of the variance of United Kingdom inflation, Econometrica 50 (1982), 987-1008.

[4] Bollersev T., Chous R.Y., Kroner K.F., ARCH modelling in finance - a review of the theory and empirical evidence, J. Econometrics 52 (1992), 5-59.

[5] Mantegna R.N., Stanley H.E., Scaling behavior in the dynamics of an economic index, Nature 376 (1995), $46-49$.

[6] de Vries D.G., Stylized facts of nominal exchange rate returns, in The Handbook of International Macroeconomics, Editor F. van der Ploeg, Blackwell, Oxford University Press, 1994.

[7] Muzy J.-F., Sornette D., Delour J., Arneodo A., Multifractal returns and hierarchical portfolio theory, Quantitative Finance 1 (2001), 131-148, cond-mat/0008069.

[8] Frank T.D., Nonlinear Fokker-Plank equations, Berlin, Springer, 2004.

[9] Shiino M., Yoshida K., Chaos-nonchaos phase transitions induced by external noise in ensembles of nonlinearly coupled oscillators, Phys. Rev. E. 63 (2001), 026210, 6 pages.

[10] Bellucci S., Trifonov A.Yu., Semiclassically-concentrated solutions for the one-dimensional Fokker-Planck equation with a nonlocal nonlinearity, J. Phys. A: Math. Gen. 38 (2005), L103-L114.

[11] Shapovalov A.V., Rezaev R.O., Trifonov A.Yu., Symmetry operators for the Fokker-Plank-Kolmogorov equation with nonlocal quadratic nonlinearity, SIGMA 3 (2007), 005, 16 pages, math-ph/0701012.

[12] Sornette D., Fokker-Planck equation of distributions of financial returns and power laws, Phys. A 290 (2001), 211-217, cond-mat/0011088.

[13] Sornette D., Critical phenomena in natural sciences. Chaos, fractals, self-organisation, and disorder: concepts and tools, Springer Series in Sinergetics, Springer-Verlag, Berlin, 2000.

[14] Gardiner C.W., Stochastic methods, Springer-Verlag, Berlin - Heidelberg - New York - Tokyo, 1985.

[15] Belov V.V., Trifonov A.Yu., Shapovalov A.V., The trajectory-coherent approximation and the system of moments for the Hartree type equation, Int. J. Math. Math. Sci. 32 (2002), 325-370, math-ph/0012046.

[16] Uhlenbeck G.E., Ornstein L.S., On the theory of the Brownian motion, Phys. Rev. 36 (1930), 823-841. 